\title{
Jackknife Empirical Likelihood Confidence Interval for the Gini Index
}

\author{
Dongliang Wang ${ }^{1}$, Yichuan Zhao*2, and Dirk W. Gilmore ${ }^{2}$ \\ ${ }^{1}$ Department of Public Health and Preventive Medicine, SUNY Upstate \\ Medical University \\ ${ }^{2}$ Department of Mathematics and Statistics, Georgia State University
}

September 29, 2015

\begin{abstract}
Jackknife empirical likelihood for the Gini index is derived. Adjusted jackknife empirical likelihood and bootstrap calibration are further investigated. The resulting interval estimators are comparable to existing empirical likelihood methods in terms of coverage accuracy, but yield much shorter intervals.
\end{abstract}

KEY WORDS: Confidence interval; Lorenz curve; Total time on test; Wilks' theorem

*Email:dz2007@gmail.com. 


\section{Introduction}

The Gini index, originated from the Gini mean difference, has been widely recognized as one of the most commonly used measures for assessing distributional inequality. The Gini index can also be expressed as the area between the 45-degree line and the Lorenz curve. The Lorenz curve, proposed by Lorenz (1905), is another commonly used measure of distributional inequality and the 45-degree line represents perfect equality.

A variety of statistical methodologies have been developed for interval estimation of the Gini index with particular focus on obtaining reliable standard error estimator with no distributional assumptions. Most recently, Davidson (2009) proposed an asymptotic approximation of the plug-in estimator of the Gini index as a sum of i.i.d. variables and derived a reliable variance estimator via delta method. The feasibility of estimating the standard error via ordinary least square regression was discussed by Ogwang (2000), Giles (2004), Modarres and Gastwirth (2006) and Giles (2006). A range of commonly used nonparametric methods have also been exploited for estimating the standard error of the Gini index estimator, such as the asymptotic theory of U-statistics by Hoeffding (1948), the jackknife method by Yitzhaki (1991) and Karagiannis (2000) and the bootstrap method by Mills and Zandvakili (1997) and Biewen (2002). In addition, parametric methods for estimating the sampling distribution of the Gini index estimator can be found in Moothathu (1985, 1990), Chotikapanich and Griffiths (2002), Abdul-Sathar et al. (2005) and Wang et al. (2015). Nonparametric variance estimation of the Gini index under more complicated sampling schemes has been discussed in Sandströmand et al. (1998) and Bhattacharya (2007).

Recently empirical likelihood methods, formally proposed by Owen (1988, 2001), were developed for interval estimation of the Gini index. The empirical likelihood ratio statistic, proposed by Qin et al. (2010), followed an asymptotic scaled asymptotic chisquare distribution, where the scale needed to be estimated in practice. Peng (2011) derived the empirical likelihood over transformed data. The transformation essentially cuts the sample size in half and the resulting interval estimator seemed to be unnecessarily 
wide.

The objective of this paper is to develop the jackknife empirical likelihood (JEL) methods for the Gini index. The rest of the paper is organized as follows. In Section 2 new JEL confidence intervals for the Gini index are proposed. In Section 3 a simulation study is carried out to compare the performance of our JEL methods with other existing methods. The proposed JEL methods are illustrated with a real data set in Section 4, followed by conclusions and discussions in Section 5 .

\section{Results}

Let $F(\cdot)=P(X \leq x)$ be the cumulative distribution function of a non-negative random variable $X$. The Gini index $G$ is formally defined as

$$
G=\frac{1}{\mu} \int_{0}^{\infty}(2 F(x)-1) x d F(x)=\frac{\mathrm{E}|X-Y|}{2 \mathrm{E} X},
$$

where $X$ and $Y$ are two independent random variables following the same distribution $F(x)$ and $\mu=\mathrm{E} X=\int_{0}^{\infty} x d F(x)$

Let $X=\left[X_{1}, X_{2}, \ldots, X_{n}\right], n \geq 2$ be i.i.d. random variables with common distribution function $F(x)$, and $X_{1: n} \leq X_{2: n} \leq \ldots \leq X_{n: n}$ be the corresponding order statistics. A commonly used nonparametric estimator for $G$ is derived as

$$
G_{n}=\frac{1}{\bar{X}} \int_{0}^{\infty} x d \hat{F}^{2}(x)-1=\frac{2}{n^{2} \bar{X}} \sum_{i=1}^{n} X_{i: n}\left(i-\frac{1}{2}\right)-1,
$$

by plugging in the empirical distribution function $\hat{F}(x)=1 / n \sum_{i=1}^{n} I\left(X_{i} \leq x\right)$, where $\bar{X}$ is the sample mean. Alternatively, the Gini index can be estimated by

$$
G_{U}=(2 \bar{X})^{-1}\left(\begin{array}{l}
n \\
2
\end{array}\right)^{-1} \sum_{1 \leq i_{1}<i_{2} \leq n}\left|X_{i}-X_{j}\right|
$$

which is a ratio of two U-statistics with the kernels $h(x, y)=|x-y|$ and $h(x)=x$, respectively. To our best knowledge, none of the existing jackknife empirical likelihood methods, including the JEL method proposed by Jing et al. (2009), can handle two U-statistics with kernels of different degrees. 


\subsection{Jackknife empirical likelihood}

We start this section by defining a new estimating equation for $G$, namely,

$$
U_{n}(\tilde{G})=\left(\begin{array}{l}
n \\
2
\end{array}\right)^{-1} \sum_{1 \leq i_{1}<i_{2} \leq n} h\left(X_{i_{1}}, X_{i_{2}} ; \tilde{G}\right)=0
$$

where $h\left(X_{i_{1}}, X_{i_{2}} ; \tilde{G}\right)=\left(X_{i_{1}}+X_{i_{2}}\right) \tilde{G}-\left|X_{i_{1}}-X_{i_{2}}\right|$. At the true value $G$ of the Gini index, $U_{n}(G)$ is in the form of a U-statistic with a symmetric kernel $h$ of degree $m=2$ and it is clear that $\mathrm{E} h\left(X_{i_{1}}, X_{i_{2}} ; G\right)=0$. The definition of $U_{n}(G)$ allows us to readily establish the Wilks' theorem of the jackknife empirical likelihood under the framework developed by Jing et al. (2009). Towards this end, the jackknife pseudo-values can be defined as

$$
\hat{V}_{i}(G)=n U_{n}(G)-(n-1) U_{n-1}^{(-i)}(G)
$$

where $U_{n-1}^{(-i)}(G)$ is the U-statistic with the $i$ th observation $X_{i}$ deleted. As indicated by Arvesen (1969), the U-statistic $U_{n}(G)$ can thus be rewritten as an average of the jackknife pseudo-values

$$
U_{n}(G)=\frac{1}{n} \sum_{i=1}^{n} \hat{V}_{i}(G)
$$

We can then follow the jackknife empirical likelihood method in Jing et al. (2009). Let $\boldsymbol{\pi}^{\prime}=\left(\pi_{1}, \ldots, \pi_{n}\right)$ be the probability vector over the jackknife pseudo-values and $H(u)=1 / n \sum_{i=1}^{n} \pi_{i} I\left\{\hat{V}_{i}(G) \leq u\right\}$ be the corresponding empirical distribution function, where $I(\cdot)$ is the indicator function. The jackknife empirical likelihood ratio at $G$ can be expressed as

$$
R(G)=\max \left\{\prod_{i=1}^{n} n \pi_{i}: \pi_{i} \geq 0, \sum_{i=1}^{n} \pi_{i}=1, \sum_{i=1}^{n} \pi_{i} \hat{V}_{i}(G)=0\right\} .
$$

By the standard Lagrange multiplier technique, the log-transformed jackknife empirical likelihood ratio at $G$ is

$$
\log R(G)=-\sum_{i=1}^{n} \log \left[1+\lambda \hat{V}_{i}(G)\right]
$$


where $\lambda=\lambda(G)$ satisfies

$$
\frac{1}{n} \sum_{i=1}^{n} \frac{\hat{V}_{i}(G)}{1+\lambda \hat{V}_{i}(G)}=0 .
$$

Let $g(x)=\operatorname{Eh}\left(x, X_{2} ; G\right)$ and $\sigma_{g}^{2}=\operatorname{var}\left(g\left(X_{1}\right)\right)$. We have Wilks' theorem for the jackknife empirical likelihood ratio statistic as below.

Theorem 2.1. Assume that $E X^{2}<\infty$ and $\sigma_{g}^{2}>0$. Then

$$
-2 \log R(G) \stackrel{d}{\rightarrow} \chi_{1}^{2} \text {, as } n \rightarrow \infty \text {. }
$$

Theorem 2.1 follows directly from Theorem 1 in Jing et al. (2009) after noting the fact that $\mathrm{E} X^{2}<\infty$ implies $E h^{2}\left(X_{1}, X_{2} ; G\right)<\infty$, by Cauchy-Schwarz inequality.

A $100(1-\alpha) \%$ JEL confidence interval for $G$ can be constructed using Theorem 2.1 as

$$
\mathbb{R}_{\alpha}=\left\{\tilde{G}:-2 \log R(\tilde{G}) \leq \chi_{1,1-\alpha}^{2}\right\}
$$

where $\chi_{1,1-\alpha}^{2}$ denotes the $(1-\alpha)$ quantile of $\chi_{1}^{2}$.

The computation for the JEL at (2.4) at a specific value $G$ is as simple as for the standard empirical likelihood ratio over the jackknife pseudo-values. The JEL interval estimator at (2.6) can be readily obtained by grid searching or using the $R$ function findUL from the package emplik, developed by Zhou and Yang (2014).

\subsection{Bootstrap calibration}

Bootstrap calibration has been commonly used to overcome the potential undercoverage problem associated with the EL confidence interval when the sample size is small. We propose the following bootstrap procedure for our JEL method.

1. Calculate $\hat{G}=G_{U}$ at $(2.2)$;

2. For $b=1, \cdots, B$.

- Generate a bootstrap sample $X^{*}=\left[X_{1}^{*}, X_{2}^{*}, \ldots, X_{n}^{*}\right]$ of size $n$ from the original data $\boldsymbol{X}=\left[X_{1}, X_{2}, \ldots, X_{n}\right]$ with replacement; 
- Calculate $\log R(\hat{G})$ at $\hat{G}$ using the equations (2.3), (2.4) and (2.5) over the bootstrapped sample $\boldsymbol{X}^{*}$.

3. Calculate the bootstrap calibrated $100(1-\alpha) \%$ JEL confidence interval for $G$ as

$$
\mathbb{R}_{\alpha}^{B}=\left\{\tilde{G}:-2 \log R(\tilde{G}) \leq c_{\alpha}^{*}\right\}
$$

where $c_{\alpha}^{*}$ is the $100(1-\alpha)$ percentile of the $B-2 \log R^{*}(\hat{G})$ values.

\subsection{Adjusted jackknife empirical likelihood}

We further adapt the adjusted empirical likelihood method, originally proposed by Chen et al. (2008) to improve the coverage probabilities by adding one more jackknife pseudovalue

$$
\hat{V}_{n+1}(G)=-\frac{a_{n}}{n} \sum_{i=1}^{n} \hat{V}_{i}(G),
$$

for some positive constant $a_{n}$. This also guarantees that the convex set of the jackknife pseudo-values contains 0 and thus the jackknife empirical likelihood is always well defined as a nonzero value.

The adjusted jackknife empirical likelihood (AJEL) ratio function after log-transformation can be expressed as

$$
\log R^{\ddagger}(G)=-\sum_{i=1}^{n+1} \log \left[1+\lambda \hat{V}_{i}(G)\right],
$$

where $\lambda=\lambda(G)$ satisfies

$$
\frac{1}{n+1} \sum_{i=1}^{n+1} \frac{\hat{V}_{i}(G)}{1+\lambda \hat{V}_{i}(G)}=0 .
$$

Generally it is recommended to take $a_{n}=\max (1, \log (n) / 2)$ by Chen et al. (2008). We state Wilks' theorem for $\log R^{\ddagger}(G)$ as below, which requires the same conditions as those in Theorem 2.1. The proof of Theorem 2.2 can be found in the Appendix.

Theorem 2.2. Assume that $E X^{2}<\infty$ and $\sigma_{g}^{2}>0$. Then

$$
-2 \log R^{\ddagger}(G) \stackrel{d}{\rightarrow} \chi_{1}^{2} \text {, as } n \rightarrow \infty
$$


Thus, a 100(1- $\alpha) \%$ AJEL confidence interval for $G$ can be constructed as follows

$$
\mathbb{R}_{\alpha}^{A}=\left\{\tilde{G}:-2 \log R^{\ddagger}(\tilde{G}) \leq \chi_{1,1-\alpha}^{2}\right\}
$$

\section{Simulation studies}

Extensive simulation studies are conducted to compare the empirical performance of the proposed JEL methods with existing methods. In order to facilitate the comparison, we rerun the simulations that were performed in Peng (2011) and focus on the improvement of the JEL methods over the EL methods. The advantage of the EL methods over other methods can be found in Qin et al. (2010) and Peng (2011).

Each of 2,000 random samples with sizes $n=20,50$ and 100 were generated from four different distributions, namely, (1) Pareto distribution $X \sim \operatorname{Pareto}(10)$ with CDF $F(x)=1-x^{-\lambda}, X>1, \lambda>1$; (2) lognormal distribution $X \sim \operatorname{lognormal}(0.5)$ in the form of $X=e^{0.5 W}$, where $W$ is a standard normal variable; (3) exponential distribution $X \sim \exp (1)$ and $(4)$ chi-square distribution $X \sim \chi_{1}^{2}$. All four distributions are commonly used in reality for modeling income data with the Gini indexes equal to 0.05, 0.28, 0.5, and 0.64, respectively. For each simulated data set, confidence intervals for the Gini index were calculated using the following methods: the EL method and bootstrap-calibrated EL method proposed in Qin et al. (2010); the EL method and bootstrap-calibrated EL method proposed in Peng (2011), the JEL method at (2.6), the AJEL method at (2.9) and the bootstrap-calibrated JEL method at (2.7), which are referred to as EL(QRW), EL(QRW)-boot, EL(Peng), EL(Peng)-boot, JEL, AJEL and JEL-boot, respectively. For methods calibrated by bootstrap, $B=4,000$ bootstrap pseudo-samples were drawn from original data with replacement.

Tables $1-4$ report the simulation results with respect to coverage probabilities and average lengths of $95 \%$ confidence intervals. Similar observations are also obtained for 90\% confidence intervals, which are available upon request. Major observations from our simulations are summarized as follows: (1) In terms of coverage probabilities, our 
JEL methods are comparable to the EL method proposed by Peng (2011), with the latter being slightly more conservative. Both methods perform obviously better than the EL(QRW) method. For Pareto(10) in Table 1, the EL(QRW) method may yield unacceptable results, particularly for small sample sizes. (2) In terms of average lengths of confidence intervals, our JEL interval estimators are significantly shorter than the EL(Peng) intervals. For instance, given data from $\exp (1)$ distribution with $n=100$ in Table 3, confidence intervals from the EL method by Peng (2011) are almost two times wider than our JEL confidence intervals. Thus our JEL methods are more accurate and powerful than the EL methods. (3) Both adjusted empirical likelihood and bootstrap calibration improve the common undercoverage problem associated with empirical likelihood, particularly for data from Pareto(10) distribution with the small sample size as shown in Table 1. Bootstrap calibration usually provides better improvement of coverage probabilities of confidence intervals, which is associated with significantly increased computational burden. For $\exp (1)$ and $\chi_{1}^{2}$ distributions as shown in Tables 3 and 4, the JEL confidence intervals calibrated by $\chi^{2}$ distribution are satisfactory and no additional adjustment is necessarily needed. We recommend interval estimators from the adjusted JEL method rather than bootstrap calibration, when the computational resource is an issue.

\section{Examples}

To illustrate the application of our JEL methods, we reanalyze a data set from the Penn World Table 5.6; see more details about the data in Heston and Summers (1991). In this paper we particularly focus on utilizing the Gini index to evaluate the dispersion of the real GDP among 134 countries in the year of 1975, 1980 and 1985, respectively. Real GDP per capita was obtained in Laspeyres index using 1985 international prices as the reference. We plot the histograms and Lorenz curves for the data at each year. As shown in Figure 1, exponential distributions seem to fit the data sets well. From the test of 
exponentiality proposed by Gail and Gastwirth (1978) utilizing the Gini statistic, the $p$ values are equal to $0.751,0.898$ and 0.695 for the year of 1975, 1980 and 1985, respectively. As shown in Table 5, the plug-in and U-statistic estimates for the Gini index at (2.1) and (2.2) are nearly 0.5, which is the Gini index of the exponential distribution. It is also worthy noting that the bias-corrected estimator in Davidson (2009) is the same as our U-statistic estimator at (2.2).

Interval estimators for the Gini index at each year are calculated using our JEL and AJEL methods and existing methods, including the nonparametric method in Davidson (2009) and the EL methods in Qin et al. (2010) and Peng (2011). The following findings can be observed in Table 5: (1) All methods yield similar confidence intervals other than

the EL method in Peng (2011), from which the intervals are wider and lifted upward; (2) All intervals cover 0.5, which is consistent with the conclusion from the tests for exponentiality that the observed data may be from an exponential distribution; (3) The intervals from Davidson's method are symmetric to the unbiased estimator at (2.2) and all EL and JEL interval estimators are right skewed.

\section{Conclusions and discussions}

In this paper we define an estimating equation for the Gini index in the form of Ustatistic and develop the jackknife empirical likelihood. We further discuss bootstrap calibration and derive the adjusted jackknife empirical likelihood by adding an extra jackknife pseudo-value. We establish the asymptotic properties of the jackknife empirical likelihood and assess the empirical performance of the resulting interval estimators. Simulation studies suggest that our JEL interval estimators are generally shorter than the empirical likelihood interval estimators while maintaining satisfactory coverage probabilities. In the future we may develop the jackknife empirical likelihood method for comparing two Gini indexes and/or for data subject to missing at random or censoring. 


\section{Acknowledgments}

We thank the Editor-in-chief Professor Paindaveine, an Associate Editor and a reviewer for their constructive comments, which significantly improve the quality of this manuscript. Yichuan Zhao was partially supported by NSA grant H98230-12-1-0209, NSF grant DMS1406163, and a RIG grant from Georgia State University.

\section{References}

Abdul-Sathar, E.I., Jeevanand, E.S. and Nair, K.R.M. (2005) "Bayesian estimation of Lorenz curve, Gini index and variance of logarithms in a Pareto distribution," Statistica LXV, 193-205.

Arvesen, J.N., (1969) "Jackknifing U-Statistics," The Annals of Mathematical Statistics 40, 2076-2100.

Bhattacharya, D. (2007) "Inference on inequality from household survey data," J. Econometrics 137, 674-707.

Biewen, M. (2002) "Bootstrap inference for inequality, mobility, and poverty measurement," Journal of Econometrics 108(2),317-342.

Chen, J., Variyath, A.M. and Abraham, B. (2008) "Adjusted Empirical Likelihood and its Properties," Journal of Computational and Graphical Statistics 17(2), 426 443.

Chotikapanich, D. and Griffiths, W.E. (2002) "Estimating Lorenz curve using a Dirichlet distribution," J. Bus. Econ. Statist. 20, 290-295.

Davidson, R. (2009) "Reliable inference for the Gini index," J. Econometrics 150, 30-40.

Gail, M.H. and Gastwirth, J.L. (1978) “A scale-free goodness-of-fit test for the exponential distribution based on the Gini statistic," J. R. Stat. Soc. Ser. B, 40-3, 
350-357.

Giles, D.E.A. (2004) "Calculating a standard error for the Gini coefficient: some further results," Oxford Bull. Econ. Statist. 66, 425-433.

Giles, D.E.A. (2006) "A cautionary note on estimating the standard error of the Gini index of inequality: Comment," Oxford Bulletin of Economics and Statistics 68, $395-396$.

Gini, C. (1912) "Variabilità e mutabilità, contributo allo studio delle distribuzioni e relazioni statistiche," Studi Economico-Giuridici della R. Università di Cagliari.

Heston, A. and Summers, R. (1991) " The Penn World Table (Mark 5): An Expanded Set of International Comparisons, 1950-1988." Quarterly Journal of Economics, $327-368$.

Hoeffding, W. (1948) "A class of statistics with asymptotically normal distribution," Annals of Mathematical Statistics 19, 293-325.

Jing, B., Yuan, J.and Zhou W. (2009) "Jackknife empirical likelihood," Journal of the American Statistical Association 104(487), 1224 - 1232.

Karagiannis, E. and Kovacevic, M. (2000) "A method to calculate the jackknife variance estimator for the Gini coefficient," Oxford Bulletin of Economics and Statistics 62 , 119-122.

Lorenz, M.O. (1905) "Methods for measuring concentration of wealth," Journal of the American Statistical Association 9, 209-219.

Mills, J.A. and Zandvakili, S. (1997) "Statistical inference via bootstrapping for measures of inequality" Journal of Applied Econometrics 12(2), 133-150.

Moothathu, T.S.K. (1985) "Distributions of maximum likelihood estimators of Lorenz curve and Gini index of exponential distribution," Ann. Inst. Statist. Math. 37, 473-479. 
Moothathu, T.S.K. (1990) "The best estimator and a strongly consistent asymptotically normal unbiased estimator of Lorenz curve, Gini index and their entropy index for Pareto distribution," Sankya, Ser. B 52, 115-127.

Modarres, R. and Gastwirth, J.L. (2006) "A cautionary note on estimating the standard error of the Gini index of inequality," Oxford Bull. Econ. Statist. 68, 385-390.

Ogwang, T. (2000) "A convenient method of computing the Gini index and its standard error," Oxford Bull. Econ. Statist. 62, 123-129.

Owen, A. (1988) "Empirical likelihood ratio confidences for single functional," Biometrika 75, 237-249.

Owen, A.B. (2001) Empirical Likelihood, Chapman and Hall/CRC press.

Peng, L. (2011) "Empirical likelihood methods for the Gini index, " Australian and New Zealand Journal of Statistics 53(2) 131-139.

Qin, Y., Rao, J.N.K. and Wu, C. (2010) "Empirical likelihood confidence intervals for the Gini measure of income inequality," Economic Modeling 27, 1429 -1435.

Sandström, A., Wretman, J.H. and Waldén, B. (1988) "Variance estimators of the Gini coefficient: probability sampling," Journal of Business and Economic Statistics 6, $113-119$.

Yitzhaki, S., (1991) "Calculating Jackknife variance estimators for parameters of the Gini method," Journal of Business \& Economic Statistics 9, 235-239.

Zhou, M. and Yang Y. (2014) "emplik: Empirical likelihood ratio for censored/truncated data." R package version 0.9-9-6. http://CRAN.R-project.org/package=emplik. 
Table 1: Coverage probabilities (average length) of 95\% confidence intervals from a variety of empirical likelihood methods; $X \sim \operatorname{Pareto}(10), G=0.05$.

\begin{tabular}{rccc}
\hline \hline & $n=20$ & $n=50$ & $n=100$ \\
\hline EL(QRW) & $0.001(0.045)$ & $0.383(0.033)$ & $0.741(0.025)$ \\
EL(QRW)-boot & $0.000(0.042)$ & $0.321(0.031)$ & $0.721(0.024)$ \\
EL(Peng) & $0.863(0.055)$ & $0.909(0.041)$ & $0.938(0.030)$ \\
EL(Peng)-boot & $0.914(0.073)$ & $0.936(0.049)$ & $0.949(0.034)$ \\
JEL & $0.844(0.047)$ & $0.908(0.035)$ & $0.921(0.026)$ \\
AJEL & $0.877(0.051)$ & $0.920(0.036)$ & $0.929(0.027)$ \\
JEL-boot & $0.891(0.071)$ & $0.938(0.046)$ & $0.939(0.030)$ \\
\hline \hline
\end{tabular}

Table 2: Coverage probabilities (average length) of 95\% confidence intervals from a variety of empirical likelihood methods; $X \sim \log$-normal(0.5), $G=0.28$.

\begin{tabular}{rccc}
\hline \hline & $n=20$ & $n=50$ & $n=100$ \\
\hline EL(QRW) & $0.869(0.153)$ & $0.922(0.105)$ & $0.939(0.077)$ \\
EL(QRW)-boot & $0.919(0.166)$ & $0.932(0.108)$ & $0.939(0.078)$ \\
EL(Peng) & $0.882(0.214)$ & $0.930(0.155)$ & $0.937(0.114)$ \\
EL(Peng)-boot & $0.949(0.276)$ & $0.950(0.171)$ & $0.947(0.120)$ \\
JEL & $0.932(0.164)$ & $0.921(0.109)$ & $0.937(0.080)$ \\
AJEL & $0.955(0.181)$ & $0.933(0.113)$ & $\mathbf{0 . 9 4 3 ( 0 . 0 8 2 )}$ \\
JEL-boot & $0.957(0.198)$ & $0.937(0.122)$ & $\mathbf{0 . 9 4 5 ( 0 . 0 8 6 )}$ \\
\hline \hline
\end{tabular}


Table 3: Coverage probabilities (average length) of 95\% confidence intervals from a variety of empirical likelihood methods; $X \sim \exp (1), G=0.50$.

\begin{tabular}{rccc}
\hline \hline & $n=20$ & $n=50$ & $n=100$ \\
\hline EL(QRW) & $0.900(0.220)$ & $0.926(0.151)$ & $0.947(0.110)$ \\
EL(QRW)-boot & $0.954(0.257)$ & $0.947(0.161)$ & $0.954(0.113)$ \\
EL(Peng) & $0.856(0.332)$ & $0.931(0.251)$ & $0.950(0.187)$ \\
EL(Peng)-boot & $0.954(0.448)$ & $0.958(0.281)$ & $0.957(0.198)$ \\
JEL & $0.936(0.261)$ & $0.940(0.159)$ & $0.951(0.112)$ \\
AJEL & $0.961(0.294)$ & $0.951(0.166)$ & $\mathbf{0 . 9 5 7 ( 0 . 1 1 5 )}$ \\
JEL-boot & $0.942(0.272)$ & $0.945(0.163)$ & $\mathbf{0 . 9 5 1 ( 0 . 1 1 5 )}$ \\
\hline \hline
\end{tabular}

Table 4: Coverage probabilities (average length) of 95\% confidence intervals from a variety of empirical likelihood methods; $X \sim \chi_{1}^{2}, G=0.64$.

\begin{tabular}{rccc}
\hline \hline & $n=20$ & $n=50$ & $n=100$ \\
\hline EL(QRW) & $0.908(0.232)$ & $0.921(0.162)$ & $0.942(0.119)$ \\
EL(QRW)-boot & $0.969(0.290)$ & $0.959(0.179)$ & $0.953(0.125)$ \\
EL(Peng) & $0.822(0.347)$ & $0.897(0.282)$ & $0.935(0.222)$ \\
EL(Peng)-boot & $0.945(0.522)$ & $0.946(0.345)$ & $0.954(0.245)$ \\
JEL & $0.952(0.288)$ & $0.937(0.172)$ & $0.946(0.122)$ \\
AJEL & $0.970(0.330)$ & $0.949(0.180)$ & $\mathbf{0 . 9 5 5 ( 0 . 1 2 5})$ \\
JEL-boot & $0.951(0.293)$ & $0.939(0.175)$ & $\mathbf{0 . 9 4 8 ( 0 . 1 2 4 )}$ \\
\hline \hline
\end{tabular}


Table 5: Point and interval estimates for the Gini indexes in the data sets.

\begin{tabular}{|c|c|c|c|c|c|}
\hline \multirow[b]{2}{*}{ Year } & \multicolumn{2}{|c|}{ Point estimate } & \multicolumn{3}{|c|}{ Interval estimate } \\
\hline & Plug-in & U-statistic & & $1-\alpha=0.90$ & $1-\alpha=0.95$ \\
\hline \multirow[t]{5}{*}{1975} & 0.488 & 0.492 & JEL & $(0.466,0.522)$ & $(0.460,0.528)$ \\
\hline & & & AJEL & $(0.465,0.522)$ & $(0.460,0.529)$ \\
\hline & & & QRW & $(0.468,0.525)$ & $(0.462,0.530)$ \\
\hline & & & Peng & $(0.467,0.599)$ & $(0.453,0.609)$ \\
\hline & & & Davidson & $(0.463,0.520)$ & $(0.459,0.526)$ \\
\hline \multirow[t]{5}{*}{1980} & 0.493 & 0.497 & JEL & $(0.470,0.527)$ & $(0.465,0.534)$ \\
\hline & & & AJEL & $(0.469,0.528)$ & $(0.464,0.535)$ \\
\hline & & & QRW & $(0.469,0.530)$ & $(0.464,0.535)$ \\
\hline & & & Peng & $(0.471,0.613)$ & $(0.455,0.625)$ \\
\hline & & & Davidson & $(0.468,0.526)$ & $(0.462,0.531)$ \\
\hline \multirow[t]{5}{*}{1985} & 0.506 & 0.510 & JEL & $(0.483,0.541)$ & $(0.478,0.547)$ \\
\hline & & & AJEL & $(0.482,0.541)$ & $(0.477,0.548)$ \\
\hline & & & QRW & $(0.484,0.541)$ & $(0.479,0.547)$ \\
\hline & & & Peng & $(0.497,0.633)$ & $(0.482,0.645)$ \\
\hline & & & Davidson & $(0.471,0.539)$ & $(0.475,0.545)$ \\
\hline
\end{tabular}


Histogram

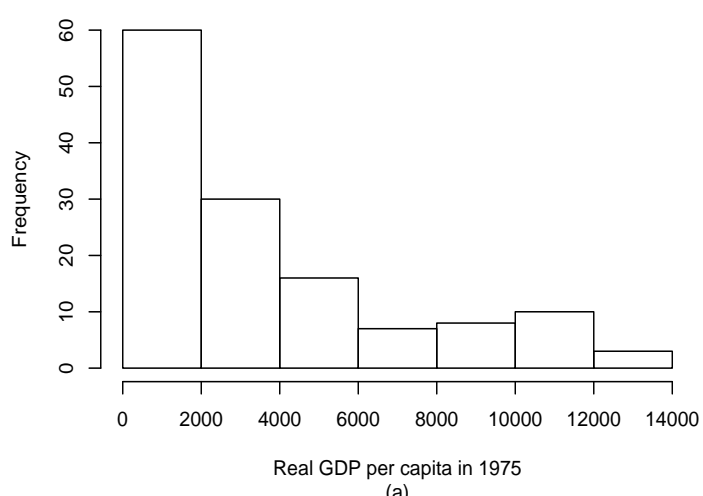

Histogram

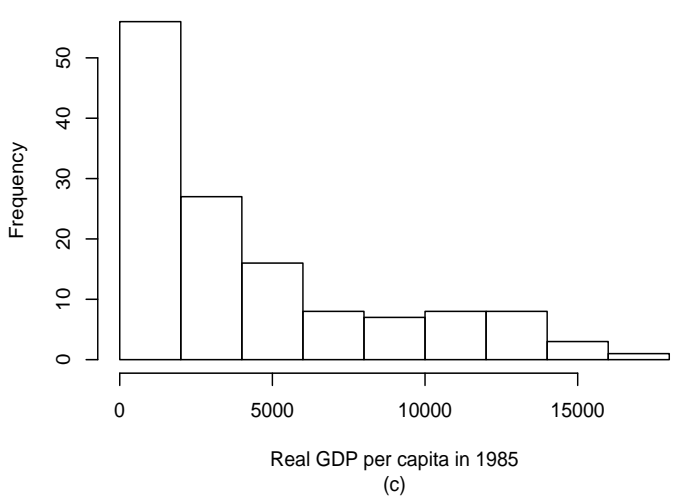

(c)

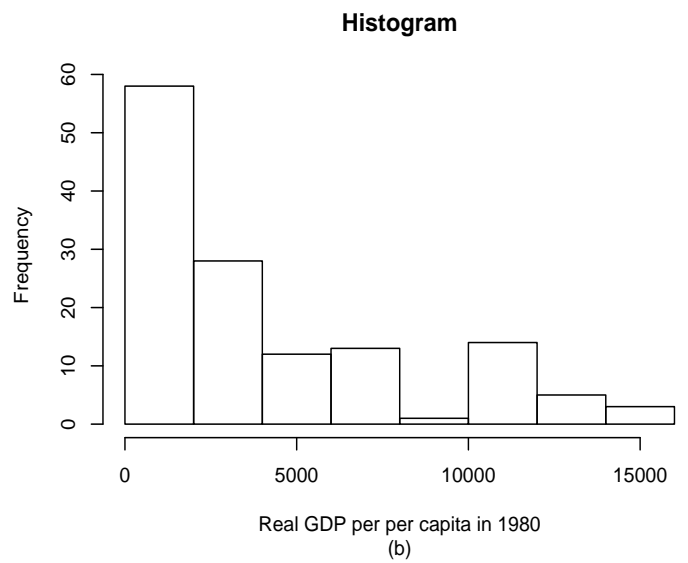

(b)

Lorenz Curves

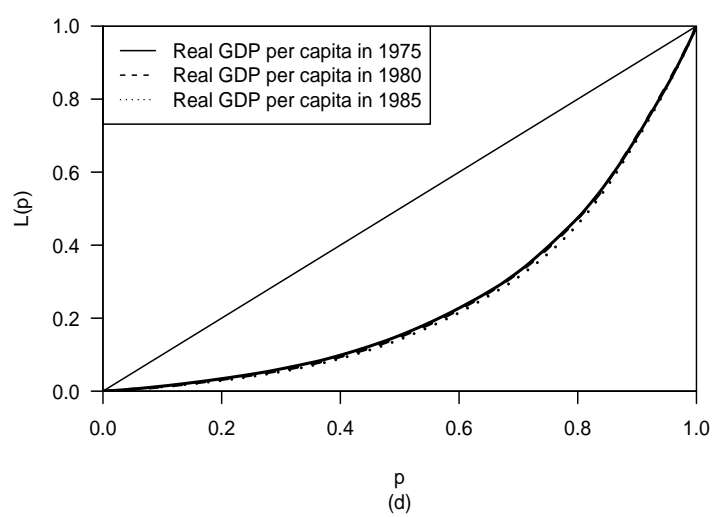

Figure 1: Related exploratory graphs for the example data sets. 


\section{Appendix: Proof of Theorem 2.2.}

The proof of Theorem 2.2 is mainly based on the lemmas and corollaries from Jing et al. (2009) with minor modifications as applied by Chen et al. (2008). Hence our proofs here will be sketchy and readers are referred to Jing et al. (2009) and Chen et al. (2008) for more details.

Firstly we show that $|\lambda|=O_{p}\left(n^{-1 / 2}\right)$. From equation (2.8) we have

$$
\begin{aligned}
0 & =\frac{1}{n}\left|\sum_{i=1}^{n+1} \hat{V}_{i}(G)-\lambda \sum_{i=1}^{n+1} \frac{\hat{V}_{i}^{2}(G)}{1+\lambda \hat{V}_{i}(G)}\right| \\
& \geq \frac{|\lambda|}{n} \sum_{i=1}^{n+1} \frac{\hat{V}_{i}^{2}(G)}{1+\lambda \hat{V}_{i}(G)}-\frac{1}{n} \sum_{i=1}^{n+1} \hat{V}_{i}(G) \\
& \geq \frac{|\lambda| S}{1+|\lambda| W_{n}}-\left|\frac{1}{n} \sum_{i=1}^{n} \hat{V}_{i}(G)\right|\left(1-a_{n} / n\right),
\end{aligned}
$$

where $S=\frac{1}{n} \sum_{i=1}^{n} \hat{V}_{i}^{2}(G)$ and $W_{n}=\max _{1 \leq i \leq n}\left|\hat{V}_{i}(G)\right|$. By Lemmas A.2 and A.3 and Corollary A.1 in Jing et al. (2009), one has $\frac{1}{n} \sum_{i=1}^{n} \hat{V}_{i}(G)=U_{n}(G)=O_{p}\left(n^{-1 / 2}\right), S=4 \sigma_{g}^{2}+o(1)$ and $W_{n}=o\left(n^{1 / 2}\right)$. Thus as long as $a_{n}=o_{p}(n)$, we have $|\lambda|=O_{p}\left(n^{-1 / 2}\right)$.

Secondly we show that $\lambda=S^{-1} U_{n}(G)+o_{p}\left(n^{-1 / 2}\right)$. From equation (2.8) it is clear that

$$
\begin{aligned}
0 & =\frac{1}{n} \sum_{i=1}^{n+1} \frac{\hat{V}_{i}(G)}{1+\lambda \hat{V}_{i}(G)} \\
& =\frac{1}{n} \sum_{i=1}^{n} \hat{V}_{i}(G)-\lambda S+o_{p}\left(n^{-1 / 2}\right)
\end{aligned}
$$

by noting that the $(n+1)$ th term of the summation is $a_{n} O_{p}\left(n^{-3 / 2}\right)$.

Finally we expand $-2 \log R^{\ddagger}(G)$ and substitute the expansion of $\lambda$.

$$
\begin{aligned}
-2 \log R^{\ddagger}(G) & =2 \sum_{i=1}^{n+1} \log \left[1+\lambda \hat{V}_{i}(G)\right] \\
& =2 \sum_{i=1}^{n+1}\left(\lambda \hat{V}_{i}(G)-\lambda^{2} \hat{V}_{i}^{2}(G) / 2\right)+o_{p}(1) \\
& =\frac{n U_{n}^{2}(G)}{S}+o_{p}(1),
\end{aligned}
$$


which converges to $\chi_{1}^{2}$ by Lemmas A.2 and A.3 from Jing et al. (2009) and Slutsky's theorem. 